Pacific

Journal of

Mathematics

NOETHER'S PROBLEM FOR DIHEDRAL 2-GROUPS II

MING-CHANG KANG

Volume $222 \quad$ No. 2

December 2005 


\title{
NOETHER'S PROBLEM FOR DIHEDRAL 2-GROUPS II
}

\author{
MING-CHANG KANG
}

\begin{abstract}
Let $K$ be any field and $G$ be a finite group. Let $G$ act on the rational function field $K\left(x_{g}: g \in G\right)$ by $K$-automorphisms defined by $g \cdot x_{h}=x_{g h}$ for any $g, h \in G$. Denote by $K(G)$ the fixed field $K\left(x_{g}: g \in G\right)^{G}$. Noether's problem asks whether $K(G)$ is rational (= purely transcendental) over $K$. A result of Serre shows that $\mathbb{Q}(G)$ is not rational when $G$ is the generalized quaternion group of order 16. We shall prove that $K(G)$ is rational over $K$ if $G$ is any nonabelian group of order 16 except when $G$ is the generalized quaternion group of order 16. When $G$ is the generalized quaternion group of order 16 and $K\left(\zeta_{8}\right)$ is a cyclic extension of $K$, then $K(G)$ is also rational over $K$.
\end{abstract}

\section{Introduction}

Let $K$ be any field and $G$ be a finite group. Let $G$ act on the rational function field $K\left(x_{g}: g \in G\right)$ by $K$-automorphisms such that $g \cdot x_{h}=x_{g h}$ for any $g, h \in G$. Denote by $K(G)$ the fixed subfield

$$
K\left(x_{g}: g \in G\right)^{G}=\left\{f \in K\left(x_{g}: g \in G\right): \sigma \cdot f=f \text { for any } \sigma \in G\right\} .
$$

Noether's problem asks whether $K(G)$ is rational (that is, purely transcendental) over $K$.

Noether's problem for finite abelian groups has been studied by Swan, Endo and Miyata, Voskresenskii, Lenstra, Colliot-Thélène and Sansuc, among others; see [Swan 1983] and the references therein. But our knowledge about Noether's problem for nonabelian groups is rather incomplete. It is known that $K(G)$ is rational if $G$ is a transitive solvable subgroup of the symmetric group $S_{p}$ when $p=$ 3, 5, 7, 11 [Furtwängler 1925], the quaternion group of order 8 [Grbner 1934], the alternating group $A_{5}$ [Maeda 1989; Kervaire and Vust 1989], $P S L_{2}$ (7), $P \operatorname{Sp}_{4}$ (3) (such that the base fields $K$ contain suitable quadratic fields of $\mathbb{Q}$ ) [Kemper 1996] or finite reflection groups [Kemper and Malle 1999]. Noether's problem for metaabelian groups and dihedral groups is discussed in [Haeuslein 1971; Hajja 1983;

MSC2000: 12F12, 13A50, 11R32, $14 \mathrm{E} 08$.

Keywords: rationality, Noether's problem, generic Galois extensions, generic polynomials, groups of order 16.

Partially supported by the National Science Council, Republic of China. 
Kang 2004]. One striking result is Saltman's Theorem [1984], which shows that $\mathbb{C}(G)$ is never rational for a $p$-group of order $p^{9}$. (See [Bogomolov 1987] for $p$ groups of smaller orders.) If $K$ is a field containing enough roots of unity, then $K(G)$ is rational for any nonabelian group of order $p^{3}$ or $p^{4}$ [Chu and Kang 2001]. A result of Serre [1995, 3.5] (see also [Garibaldi et al. 2003, Theorem 33.26 and Example 33.27, pp. 89-90]) shows that $\mathbb{Q}(G)$ is not rational if $G$ is the generalized quaternion group of order 16 (see Theorem 1.3 for the definition of this group); in fact, it is shown that, if $G$ is a finite group whose 2-Sylow subgroup is isomorphic to the generalized quaternion group, then $\mathbb{Q}(G)$ is not rational [Garibaldi et al. 2003, Theorem 34.7, p. 92]. Thus it would be interesting to investigate for which fields $K$ and 2-groups $G$ the field $K(G)$ will be rational, at least for groups of small order. It turns out that, if $G$ is a nonabelian group of order 8 or 16, Serre's counterexample is the only exceptional case. See Theorem 1.3.

One motivation to study Noether's problem arises from the inverse Galois problem, in particular, the existence of a generic polynomial for $G$-extensions over $K$ (equivalently, the existence of a generic Galois $G$-extension over $K$ ). If $K$ is an infinite field and $K(G)$ is rational over $K$, there exists a generic polynomial for $G$ extensions over $K$ [Saltman 1982, Theorem 5.1; DeMeyer and McKenzie 2003]. (See also [Hashimoto and Miyake 1999] for the case of dihedral extensions.) For most $p$-groups $G$, it is still unknown whether a generic Galois $G$-extension over $K$ exists [Saltman 1982]. We just mention some relevant results:

Theorem 1.1. Let $K$ be any infinite field.

(1) [Black 1999] There exists a generic Galois $G$-extension over $K$ if $G=D_{4}$ or $D_{8}$, where $D_{4}$ and $D_{8}$ are the dihedral groups of order 8 and 16 .

(2) [Ledet 2000; 2001] There exists a generic polynomial for G-extensions over $K$, if $G$ is

(i) a nonabelian group of order 8 , or

(ii) a nonabelian group of order 16 defined by

$$
\begin{aligned}
& G=\left\langle\sigma, \tau: \sigma^{8}=\tau^{2}=1, \tau^{-1} \sigma \tau=\sigma^{a}\right\rangle \quad \text { with } a=3,5,7, \text { or } \\
& G=\left\langle\sigma, \tau, \lambda: \sigma^{4}=\tau^{2}=\lambda^{2}=1, \tau^{-1} \sigma \tau=\lambda^{-1} \sigma \lambda=\sigma, \lambda^{-1} \tau \lambda=\sigma^{2} \tau\right\rangle .
\end{aligned}
$$

Theorem 1.2 [Chu et al. 2004]. For any field $K, K(G)$ is rational over $K$ provided that $G$ is

(i) a nonabelian group of order 8 , or

(ii) a nonabelian group of order 16 defined by

$$
G=\left\langle\sigma, \tau: \sigma^{8}=\tau^{2}=1, \tau^{-1} \sigma \tau=\sigma^{a}\right\rangle \quad \text { with } a=3,5 \text { or } 7 .
$$


What we will prove in this article completes our knowledge of Noether's problem for groups of order 16:

Theorem 1.3. For any field $K, K(G)$ is rational over $K$, if $G$ is any nonabelian group of order 16 except possibly the generalized quaternion group defined by

$$
G=\left\langle\sigma, \tau: \sigma^{8}=\tau^{4}=1, \sigma^{4}=\tau^{2}, \tau^{-1} \sigma \tau=\sigma^{-1}\right\rangle .
$$

For this "exceptional" group $G$, if $K\left(\zeta_{8}\right)$ is cyclic over $K$ where $\zeta_{8}$ is a primitive 8 -th root of unity (in case char $K \neq 2$ ), then $K(G)$ is rational also.

As mentioned before we cannot improve the "exceptional" group $G$ in Theorem 1.3 if $K=\mathbb{Q}$ because of Serre's Theorem. We will remark that the novelty of Theorem 1.3. is that no "unnecessary" restriction on the field is assumed; it is known that $K(G)$ is always rational provided that $G$ is any nonabelian $p$-group of order $p^{3}$ or $p^{4}$ with exponent $p^{e}$ and $K$ is a field containing a primitive $p^{e}$-th root of unity [Chu and Kang 2001, Theorem 1.6].

As an application of the above theorem we obtain the following theorem, thanks to [Saltman 1982, Theorem 5.1].

Theorem 1.4. For any infinite field $K$, a generic Galois $G$-extension over $K$ exists for any nonabelian group $G$ of order 16 , except possibly the generalized quaternion group of order 16 defined in Theorem 1.3. If $G$ is the generalized quaternion group of order 16 and $K\left(\zeta_{8}\right)$ is cyclic over $K$ (in case char $K \neq 2$ ), then a generic Galois $G$-extension over $K$ exists also.

We shall organize this paper as follows. We will recall some preliminaries in Section 2. Theorem 1.3 will be proved in Section 3. We will remark that, since the proof of this theorem is constructive, a transcendental basis of $K(G)$ can be exhibited explicitly. Thus a generic polynomial for $G$-extensions over $K$ can be found by applying [Kemper and Malle 1999, Proposition 3.1]. Since Noether's problem for finite abelian groups was completely solved by Lenstra [1974], we will concentrate on nonabelian groups.

Notations and terminologies. A field extension $L$ over $K$ is rational if $L$ is purely transcendental over $K ; L$ is called stably rational over $K$ if there exist elements $y_{1}, \ldots, y_{N}$ which are algebraically independent over $L$ such that $L\left(y_{1}, \ldots, y_{N}\right)$ is rational over $K$. $\zeta_{n}$ will denote a primitive $n$-th root of unity in some extension field of the field $K$ when char $K=0$ or char $K=p>0$ with $p \nmid n$. Finally, recall the definition $K(G)$ at the beginning of this section: $K(G)=K\left(x_{g}: g \in G\right)^{G}$. The representation space of the regular representation of $G$ over $K$ is denoted by $W=\bigoplus_{g \in G} K \cdot x(g)$ where $G$ acts on $W$ by $g \cdot x(h)=x(g h)$ for any $g, h \in G$. 


\section{Generalities}

We recall a variant of Hilbert's Theorem 90 that has been used by many people under different guises.

Theorem 2.1 [Hajja and Kang 1995, Theorem 1]. Let $L$ be a field and $G$ a finite group acting on $L\left(x_{1}, \ldots, x_{m}\right)$, the rational function field of $m$ variables over $L$. Suppose that

(i) for any $\sigma \in G, \sigma(L) \subset L$;

(ii) the restriction of the action of $G$ to $L$ is faithful;

(iii) for any $\sigma \in G$,

$$
\left(\begin{array}{c}
\sigma\left(x_{1}\right) \\
\cdot \\
\cdot \\
\cdot \\
\sigma\left(x_{m}\right)
\end{array}\right)=A(\sigma)\left(\begin{array}{c}
x_{1} \\
\cdot \\
\cdot \\
\cdot \\
x_{m}
\end{array}\right)+B(\sigma)
$$

where $A(\sigma) \in \mathrm{GL}_{m}(L)$ and $B(\sigma)$ is an $m \times 1$ matrix over $L$.

Then there exist $z_{1}, \ldots, z_{m} \in L\left(x_{1}, \ldots, x_{m}\right)$ such that

$$
L\left(x_{1}, \ldots, x_{m}\right)^{G}=L^{G}\left(z_{1}, \ldots, z_{m}\right)
$$

and $\sigma\left(z_{i}\right)=z_{i}$ for any $\sigma \in G$ and any $1 \leq i \leq m$.

Theorem 2.2 [Hajja and Kang 1994, Lemma (2.7)]. Let $K$ be any field, $a, b \in$ $K \backslash\{0\}$ and $\sigma: K(x, y) \rightarrow K(x, y)$ the $K$-automorphism defined by $\sigma(x)=a / x$, $\sigma(y)=b / y$. Then $K(x, y)^{\langle\sigma\rangle}=K(u, v)$ where

$$
u=\frac{x-\frac{a}{x}}{x y-\frac{a b}{x y}}, \quad v=\frac{y-\frac{b}{y}}{x y-\frac{a b}{x y}} .
$$

Moreover, $x+(a / x)=\left(-b u^{2}+a v^{2}+1\right) / v, y+(b / y)=\left(b u^{2}-a v^{2}+1\right) / u$, $x y+(a b /(x y))=\left(-b u^{2}-a v^{2}+1\right) /(u v)$.

Theorem 2.3 [Kuniyoshi 1955; Miyata 1971]. Let $K$ be a field with char $K=$ $p>0$ and $G$ be a p-group. Then $K(V)^{G}$ is rational over $K$ for any representation $\rho: G \rightarrow \mathrm{GL}(V)$ where $V$ is a finite-dimensional vector space over $K$.

Proof. Since char $K=p>0$ and $|G|=p^{m}$, any representation of $G$ can be triangulated. Apply [Hajja and Kang 1994, Theorem (2.2)]. 


\section{Proof of Theorem 1.3}

Without loss of generality we will assume that $K$ is any field with char $K \neq 2$ throughout this section, because Theorem 2.3 will take care of the case char $K=2$.

Here is a list of nonabelian groups of order 16, which can be found in [Huppert 1967, p. 349] or in [Chu and Kang 2001, Theorem 3.4]:

(I) $\left\langle\sigma, \tau: \sigma^{8}=\tau^{2}=1, \tau^{-1} \sigma \tau=\sigma^{-1}\right\rangle$,

(II) $\left\langle\sigma, \tau: \sigma^{8}=\tau^{4}=1, \sigma^{4}=\tau^{2}, \tau^{-1} \sigma \tau=\sigma^{-1}\right\rangle$,

(III) $\left\langle\sigma, \tau: \sigma^{8}=\tau^{2}=1, \tau^{-1} \sigma \tau=\sigma^{5}\right\rangle$,

(IV) $\left\langle\sigma, \tau: \sigma^{8}=\tau^{2}=1, \tau^{-1} \sigma \tau=\sigma^{3}\right\rangle$,

(V) $\left\langle\sigma, \tau, \lambda: \sigma^{4}=\tau^{2}=\lambda^{2}=1, \tau^{-1} \sigma \tau=\lambda^{-1} \sigma \lambda=\sigma, \lambda^{-1} \tau \lambda=\sigma^{2} \tau\right\rangle$,

(VI) $\left\langle\sigma, \tau, \lambda: \sigma^{4}=\tau^{2}=\lambda^{2}=1, \tau^{-1} \sigma \tau=\sigma^{-1}, \lambda^{-1} \sigma \lambda=\sigma, \lambda^{-1} \tau \lambda=\tau\right\rangle$,

(VII) $\left\langle\sigma, \tau, \lambda: \sigma^{4}=\tau^{4}=\lambda^{2}=1, \sigma^{2}=\tau^{2}, \tau^{-1} \sigma \tau=\sigma^{-1}, \lambda^{-1} \sigma \lambda=\sigma, \lambda^{-1} \tau \lambda=\tau\right\rangle$,

(VIII) $\left\langle\sigma, \tau: \sigma^{4}=\tau^{4}=1, \tau^{-1} \sigma \tau=\sigma^{-1}\right\rangle$,

(IX) $\left\langle\sigma, \tau, \lambda: \sigma^{4}=\tau^{2}=\lambda^{2}=1, \tau^{-1} \sigma \tau=\sigma, \lambda^{-1} \sigma \lambda=\sigma \tau, \lambda^{-1} \tau \lambda=\tau\right\rangle$.

Because we have solved the rationality problem for the groups (I), (III), (IV) in [Chu et al. 2004], we will consider the remaining six groups in this article.

Case 1. The group (V): $G=\left\langle\sigma, \tau, \lambda: \sigma^{4}=\tau^{2}=\lambda^{2}=1, \tau^{-1} \sigma \tau=\lambda^{-1} \sigma \lambda=\right.$ $\left.\sigma, \lambda^{-1} \tau \lambda=\sigma^{2} \tau\right\rangle$.

If $\sqrt{-1} \in K$, then $K(G)$ is rational by [Chu and Kang 2001, Theorem 1.6]. Hence we shall assume that $\sqrt{-1} \notin K$ from now on.

Let $W=\bigoplus_{g \in K} K \cdot x(g)$ be the representation space of the regular representation of $G$. Define

$$
\begin{aligned}
& x_{1}=x(1)+x(\tau)-x\left(\sigma^{2}\right)-x\left(\sigma^{2} \tau\right), \\
& x_{2}=\sigma \cdot x_{1}, \quad x_{3}=\lambda \cdot x_{1}, \quad x_{4}=\lambda \sigma \cdot x_{1} .
\end{aligned}
$$

Then we find that

$$
\begin{aligned}
& \sigma: x_{1} \mapsto x_{2} \mapsto-x_{1}, \quad x_{3} \mapsto x_{4} \mapsto-x_{3}, \\
& \tau: x_{1} \mapsto x_{1}, \quad x_{2} \mapsto x_{2}, \quad x_{3} \mapsto-x_{3}, \quad x_{4} \mapsto-x_{4}, \\
& \lambda: x_{1} \leftrightarrow x_{3}, \quad x_{2} \leftrightarrow x_{4} .
\end{aligned}
$$

Moreover, $\bigoplus_{1 \leq i \leq 4} K \cdot x_{i}$ is a faithful $G$-subspace of $W$. Thus $K(G)$ is rational if $K\left(x_{1}, \ldots, x_{4}\right)^{G}$ is rational by Theorem 2.1.

Let $\operatorname{Gal}(K \sqrt{-1} / K)=\langle\rho\rangle$ and $\rho: \sqrt{-1} \mapsto-\sqrt{-1}$. We extend the actions of $\sigma, \tau, \lambda, \rho$ to $K(\sqrt{-1})\left(x_{1}, \ldots, x_{4}\right)$ by requiring $\sigma(\sqrt{-1})=\tau(\sqrt{-1})=\lambda(\sqrt{-1})=$ 
$\sqrt{-1}, \rho\left(x_{i}\right)=x_{i}$ for $1 \leq i \leq 4$. Then

$$
\begin{aligned}
K\left(x_{1}, \ldots, x_{4}\right)^{\langle\sigma, \tau, \lambda\rangle} & =\left\{K(\sqrt{-1})\left(x_{1}, \ldots, x_{4}\right)^{\langle\rho\rangle}\right\}^{\langle\sigma, \tau, \lambda\rangle} \\
& =K(\sqrt{-1})\left(x_{1}, \ldots, x_{4}\right)^{\langle\sigma, \tau, \lambda, \rho\rangle} .
\end{aligned}
$$

Define

$$
\begin{array}{ll}
y_{1}=\sqrt{-1} x_{1}+x_{2}, & y_{2}=-\sqrt{-1} x_{1}+x_{2}, \\
y_{3}=\sqrt{-1} x_{3}+x_{4}, & y_{4}=-\sqrt{-1} x_{3}+x_{4} .
\end{array}
$$

Then we get

$$
\begin{aligned}
& \sigma: y_{1} \mapsto \sqrt{-1} y_{1}, \quad y_{2} \mapsto-\sqrt{-1} y_{2}, \quad y_{3} \mapsto \sqrt{-1} y_{3}, \quad y_{4} \mapsto-\sqrt{-1} y_{4}, \\
& \tau: y_{1} \mapsto y_{1}, \quad y_{2} \mapsto y_{2}, \quad y_{3} \mapsto-y_{3}, \quad y_{4} \mapsto-y_{4}, \\
& \lambda: y_{1} \leftrightarrow y_{3}, \quad y_{2} \leftrightarrow y_{4}, \\
& \rho: y_{1} \leftrightarrow y_{2}, \quad y_{3} \leftrightarrow y_{4} .
\end{aligned}
$$

Define

$$
z_{1}=y_{1} y_{2}, \quad z_{2}=y_{3} y_{4}, \quad z_{3}=y_{3} / y_{1}, \quad z_{4}=y_{1}^{4} .
$$

Then $K(\sqrt{-1})\left(y_{1}, \ldots, y_{4}\right)^{<\sigma>}=K(\sqrt{-1})\left(z_{1}, \ldots, z_{4}\right)$; moreover,

$$
\begin{aligned}
& \tau: z_{1} \mapsto z_{1}, \quad z_{2} \mapsto z_{2}, \quad z_{3} \mapsto-z_{3}, \quad z_{4} \mapsto z_{4}, \\
& \lambda: z_{1} \leftrightarrow z_{2}, \quad z_{3} \mapsto 1 / z_{3}, \quad z_{4} \mapsto z_{3}^{4} z_{4}, \\
& \rho: z_{1} \mapsto z_{1}, \quad z_{2} \mapsto z_{2}, \quad z_{3} \mapsto z_{2} /\left(z_{1} z_{3}\right), \quad z_{4} \mapsto z_{1}^{4} / z_{4} .
\end{aligned}
$$

Thus $K(\sqrt{-1})\left(z_{1}, \ldots, z_{4}\right)^{\langle\tau\rangle}=K(\sqrt{-1})\left(z_{1}, z_{2}, z_{3}^{2}, z_{4}\right)$.

Define

$$
u_{1}=z_{1} z_{2}, \quad u_{2}=z_{3}^{2} z_{4} /\left(z_{1} z_{2}\right), \quad x=z_{1}, \quad y=z_{1} z_{3}^{2} / z_{2} .
$$

Then we find that

$$
\begin{aligned}
& \lambda: u_{1} \mapsto u_{1}, \quad u_{2} \mapsto u_{2}, \quad x \mapsto a / x, \quad y \mapsto b / y, \\
& \rho: u_{1} \mapsto u_{1}, \quad u_{2} \mapsto 1 / u_{2}, \quad x \mapsto x, \quad y \mapsto 1 / y .
\end{aligned}
$$

where $a=u_{1}, b=1$.

Define

$$
u=\frac{x-\frac{a}{x}}{x y-\frac{a b}{x y}}, \quad v=\frac{y-\frac{b}{y}}{x y-\frac{a b}{x y}} .
$$

By Theorem 2.2, $K(\sqrt{-1})\left(u_{1}, u_{2}, x, y\right)^{\langle\lambda\rangle}=K(\sqrt{-1})\left(u_{1}, u_{2}, u, v\right)$. 
It is routine to check that

$$
\rho: u \mapsto \frac{x-\frac{a}{x}}{\frac{b x}{y}-\frac{a y}{x}}, \quad v \mapsto-\frac{y-\frac{b}{y}}{\frac{b x}{y}-\frac{a y}{x}} .
$$

Define $w=u / v$. Then $\rho(w)=-w$. It is not difficult to verify that

$$
\frac{x-\frac{a}{x}}{\frac{b x}{y}-\frac{a y}{x}}=\frac{u}{b u^{2}-a v^{2}} .
$$

In fact, using Theorem 2.2, the right-hand side of (3-1) is equal to $(y+(b / y)-$ $(1 / u))^{-1}$. It is very easy to check that the left-hand side of (3-1) is equal to the same quantity.

It follows that $\rho(u)=u /\left(b u^{2}-a v^{2}\right)=c / u$ where $c=w^{2} /\left(b w^{2}-a\right)$.

Define

$$
t=u_{1}, \quad s=\sqrt{-1} w, \quad q=w\left(1+u_{2}\right) /\left(1-u_{2}\right) .
$$

Then

$$
\rho: \sqrt{-1} \mapsto-\sqrt{-1}, \quad t \mapsto t, \quad s \mapsto s, \quad q \mapsto q, \quad u \mapsto c / u
$$

where $c=w^{2} /\left(b w^{2}-a\right)=s^{2} /\left(s^{2}+t\right)$.

Define $p=\left(s^{2}+t\right) u / s$. Then $\rho(p)=A / p$ where $A=s^{2}+t$.

It follows that $K(\sqrt{-1})\left(u_{1}, u_{2}, u, v\right)^{\langle\rho\rangle}=K(\sqrt{-1})(t, s, p, q)^{\langle\rho\rangle}=K(\sqrt{-1})$ $(t, s, p)^{\langle\rho\rangle}(q)=K(t, s, X, Y, q)$ where $X=p+(A / p), Y=\sqrt{-1}(p-(A / p))$. Note that a relation of $X$ and $Y$ is

$$
X^{2}+Y^{2}=4 A=4\left(s^{2}+t\right)
$$

Hence $t \in K(s, X, Y)$. It follows that $K(t, s, X, Y, q)=K(s, X, Y, q)$ is rational over $K$.

Case 2. The group (VI): $G=\left\langle\sigma, \tau, \lambda: \sigma^{4}=\tau^{2}=\lambda^{2}=1, \tau^{-1} \sigma \tau=\sigma^{-1}, \lambda^{-1} \sigma \lambda=\right.$ $\left.\sigma, \lambda^{-1} \tau \lambda=\tau\right\rangle$.

As before, let $W=\bigoplus_{g \in G} K \cdot x(g)$ be the regular representation of $G$. Define

$$
\begin{aligned}
& x_{1}=x(1)+x(\tau)-x\left(\sigma^{2}\right)-x\left(\sigma^{2} \tau\right), \\
& x_{2}=\sigma \cdot x_{1}, \quad x_{3}=\lambda \cdot x_{1}, \quad x_{4}=\lambda \sigma \cdot x_{1} .
\end{aligned}
$$


Then we find that

$$
\begin{aligned}
& \sigma: x_{1} \mapsto x_{2} \mapsto-x_{1}, \quad x_{3} \mapsto x_{4} \mapsto-x_{3}, \\
& \tau: x_{1} \mapsto x_{1}, \quad x_{2} \mapsto-x_{2}, \quad x_{3} \mapsto x_{3}, \quad x_{4} \mapsto-x_{4}, \\
& \lambda: x_{1} \leftrightarrow x_{3}, \quad x_{2} \leftrightarrow x_{4} .
\end{aligned}
$$

As in Case 1 , it suffices to consider the case $\sqrt{-1} \notin K$. Let $\operatorname{Gal}(K(\sqrt{-1} / K)$ $=\langle\rho\rangle$ with $\rho(\sqrt{-1})=-\sqrt{-1}$.

Define $y_{i}$ and $z_{i}$, for $1 \leq i \leq 4$, the same way as in Case 1 . We find

$$
K(\sqrt{-1})\left(x_{1}, \ldots, x_{4}\right)^{\langle\sigma\rangle}=K(\sqrt{-1})\left(z_{1}, \ldots, z_{4}\right) .
$$

The actions of $\lambda$ and $\rho$ on $z_{1}, \ldots, z_{4}$ are the same as in Case 1 , while $\tau \rho(\sqrt{-1})=$ $-\sqrt{-1}, \tau \rho\left(z_{i}\right)=z_{i}$ for $1 \leq i \leq 4$. Thus

$$
K(\sqrt{-1})\left(z_{1}, \ldots, z_{4}\right)^{\langle\tau, \lambda, \rho\rangle}=K(\sqrt{-1})\left(z_{1}, \ldots, z_{4}\right)^{\langle\tau \rho, \lambda, \rho\rangle}=K\left(z_{1}, \ldots, z_{4}\right)^{\langle\lambda, \rho\rangle} .
$$

Define

$$
u_{1}=z_{1} z_{2}, \quad u_{2}=z_{1}, \quad x=z_{3} / z_{2}, \quad y=z_{3}^{2} z_{4} /\left(z_{1} z_{2}\right) .
$$

We find that

$$
\begin{aligned}
& \lambda ; u_{1} \mapsto u_{1}, \quad u_{2} \mapsto u_{1} / u_{2}, \quad x \mapsto a / x, \quad y \mapsto y, \\
& \rho: u_{1} \mapsto u_{1}, \quad u_{2} \mapsto u_{2}, \quad x \mapsto a / x, \quad y \mapsto b / y,
\end{aligned}
$$

where $a=1 / u_{1}$ and $b=1$.

Define $u, v$, and $w=u / v$ the same way as in Case 1 . Then $K\left(u_{1}, u_{2}, x, y\right)^{\langle\rho\rangle}=$ $K\left(u_{1}, u_{2}, w, u\right)$ and we find that

$$
\lambda: u_{1} \mapsto u_{1}, \quad u_{2} \mapsto u_{1} / u_{2}, \quad w \mapsto-w, \quad u \mapsto c / u,
$$

where $c=w^{2} /\left(b w^{2}-a\right)$.

Define

$$
w_{1}=-1 /\left(u_{1} w^{2}\right), \quad w_{2}=w w_{1} u_{2}, \quad w_{3}=u / c .
$$

Then $K\left(u_{1}, u_{2}, w, u\right)=K\left(w_{1}, w_{2}, w_{3}, w\right)$ and

$$
\lambda: w_{1} \mapsto w_{1}, \quad w_{2} \mapsto w_{1} / w_{2}, \quad w_{3} \mapsto\left(w_{1}+1\right) / w_{3} .
$$

By Theorem 2.1, it suffices to show that $K\left(w_{1}, w_{2}, w_{3}\right)^{\langle\lambda\rangle}$ is rational over $K$. But this is easy because it is even rational over $K\left(w_{1}\right)$ by Theorem 2.2.

Case 3. The group (VII): $G=\left\langle\sigma, \tau, \lambda: \sigma^{4}=\tau^{4}=\lambda^{2}=1, \sigma^{2}=\tau^{2}, \tau^{-1} \sigma \tau=\right.$ $\left.\sigma^{-1}, \lambda^{-1} \sigma \lambda=\sigma, \lambda^{-1} \tau \lambda=\tau\right\rangle$. 
Define a faithful $G$-subspace $\bigoplus_{1 \leq i \leq 5} K \cdot x_{i}$ in the representation space $W=$ $\bigoplus_{g \in G} K \cdot x(g)$ of the regular representation by

$$
\begin{aligned}
& x_{1}=x(1)+x(\lambda)-x\left(\sigma^{2}\right)-x\left(\sigma^{2} \lambda\right), \\
& x_{2}=\sigma \cdot x_{1}, x_{3}=\tau \cdot x_{1}, x_{4}=\tau \sigma \cdot x_{1}, \\
& x_{5}=\sum_{h \in H} x(h)-\sum_{h \in H} x(h \lambda)
\end{aligned}
$$

where $H$ is the subgroup generated by $\sigma$ and $\tau$.

We find that

$$
\begin{aligned}
& \sigma: x_{1} \mapsto x_{2} \mapsto-x_{1}, \quad x_{3} \mapsto-x_{4}, \quad x_{4} \mapsto x_{3}, \quad x_{5} \mapsto x_{5}, \\
& \tau: x_{1} \mapsto x_{3}, \quad x_{2} \mapsto x_{4}, \quad x_{3} \mapsto-x_{1}, \quad x_{4} \mapsto-x_{2}, \quad x_{5} \mapsto x_{5}, \\
& \lambda: x_{1} \mapsto x_{1}, \quad x_{2} \mapsto x_{2}, \quad x_{3} \mapsto x_{3}, \quad x_{4} \mapsto x_{4}, \quad x_{5} \mapsto-x_{5} .
\end{aligned}
$$

Thus

$$
K\left(x_{1}, \ldots, x_{5}\right)^{\langle\lambda\rangle}=K\left(x_{1}, \ldots, x_{5}^{2}\right), \quad K\left(x_{1}, \ldots, x_{5}\right)^{\langle\sigma, \tau, \lambda\rangle}=K\left(x_{1}, \ldots, x_{4}\right)^{\langle\sigma, \tau\rangle}\left(x_{5}^{2}\right) .
$$

However, the fixed field $K\left(x_{1}, \ldots, x_{4}\right)^{\langle\sigma, \tau\rangle}$ is exactly the same as in the proof of [Chu et al. 2004, Theorem 2.6]. (The subgroup $H=\langle\sigma, \tau\rangle$ is the quaternion group of order 8.) Hence the result.

Case 4. The group (VIII): $G=\left\langle\sigma, \tau: \sigma^{4}=\tau^{4}=1, \tau^{-1} \sigma \tau=\sigma^{-1}\right\rangle$.

Define a faithful $G$-subspace $\bigoplus_{1 \leq i \leq 4} K \cdot x_{i}$ in $W=\bigoplus_{g \in G} K \cdot x(g)$ by

$$
\begin{aligned}
& x_{1}=x(1)+x(\tau)-x\left(\sigma^{2}\right)-x\left(\sigma^{2} \tau\right), \quad x_{2}=\sigma \cdot x_{1}, \\
& x_{3}=\sum_{0 \leq i \leq 3} x\left(\sigma^{i}\right)-\sum_{0 \leq i \leq 3} x\left(\sigma^{i} \tau^{2}\right), \quad x_{4}=\tau \cdot x_{3} .
\end{aligned}
$$

Then we find

$$
\begin{aligned}
& \sigma: x_{1} \mapsto x_{2} \mapsto-x_{1}, \quad x_{3} \mapsto x_{3}, \quad x_{4} \mapsto x_{4}, \\
& \tau: x_{1} \mapsto x_{1}, \quad x_{2} \mapsto-x_{2}, \quad x_{3} \mapsto x_{4} \mapsto-x_{3} .
\end{aligned}
$$

It is clear that $K\left(x_{1}, \ldots, x_{4}\right)^{\left\langle\sigma^{2}, \tau^{2}\right\rangle}=K\left(y_{1}, \ldots, y_{4}\right)$ where $y_{1}=x_{1} x_{2}, y_{2}=$ $x_{1}^{2}, y_{3}=x_{3} x_{4}, y_{4}=x_{3}^{2}$.

Note that

$$
\begin{aligned}
& \sigma: y_{1} \mapsto-y_{1}, \quad y_{2} \mapsto y_{1}^{2} / y_{2}, \quad y_{3} \mapsto y_{3}, \quad y_{4} \mapsto y_{4}, \\
& \tau: y_{1} \mapsto-y_{1}, \quad y_{2} \mapsto y_{2}, \quad y_{3} \mapsto-y_{3}, \quad y_{4} \mapsto y_{3}^{2} / y_{4} .
\end{aligned}
$$

Define $u_{1}=y_{2}+\left(y_{1}^{2} / y_{2}\right), u_{2}=\left(y_{1} / y_{2}\right)-\left(y_{2} / y_{1}\right), u_{3}=y_{3}, u_{4}=y_{3} / y_{4}$. Since $\left[K\left(u_{1}, u_{2}\right)\left(y_{1}\right): K\left(u_{1}, u_{2}\right)\right] \leq 2$, it follows that $K\left(y_{1}, \ldots, y_{4}\right)^{\langle\sigma\rangle}=K\left(u_{1}, \ldots, u_{4}\right)$. 
Note that

$$
\tau: u_{1} \mapsto u_{1}, \quad u_{2} \mapsto-u_{2}, \quad u_{3} \mapsto-u_{3}, \quad u_{4} \mapsto-1 / u_{4}
$$

Hence $K\left(u_{1}, \ldots, u_{4}\right)^{\langle\tau\rangle}=K\left(u_{1}, u_{2} u_{3}, u_{3} u_{4}+\left(u_{3} / u_{4}\right), u_{4}-\left(1 / u_{4}\right)\right)$ is rational over $K$.

Case 5. The group (IX): $G=\left\langle\sigma, \tau, \lambda: \sigma^{4}=\tau^{2}=\lambda^{2}=1, \tau^{-1} \sigma \tau=\sigma, \lambda^{-1} \sigma \lambda=\right.$ $\left.\sigma \tau, \lambda^{-1} \tau \lambda=\tau\right\rangle$

Define a faithful $G$-subspace $\bigoplus_{1 \leq i \leq 4} K \cdot x_{i}$ in $W=\bigoplus_{g \in G} K \cdot x(g)$ by

$$
\begin{aligned}
& x_{1}=x(1)-x\left(\sigma^{2}\right)-x(\lambda)+x\left(\sigma^{2} \lambda\right), \\
& x_{2}=\sigma \cdot x_{1}, \quad x_{3}=\tau \cdot x_{1}, \quad x_{4}=\tau \sigma \cdot x_{1} .
\end{aligned}
$$

Then we find that

$$
\begin{aligned}
& \sigma: x_{1} \mapsto x_{2} \mapsto-x_{1}, \quad x_{3} \mapsto x_{4} \mapsto-x_{3}, \\
& \tau: x_{1} \leftrightarrow x_{3}, \quad x_{2} \leftrightarrow x_{4}, \\
& \lambda: x_{1} \mapsto-x_{1}, \quad x_{2} \mapsto-x_{4}, \quad x_{3} \mapsto-x_{3}, \quad x_{4} \mapsto-x_{2} .
\end{aligned}
$$

Define

$$
y_{1}=x_{1}-x_{3}, \quad y_{2}=x_{2}-x_{4}, \quad y_{3}=x_{1}+x_{3}, \quad y_{4}=x_{2}+x_{4} .
$$

It follows that

$$
\begin{aligned}
& \sigma: y_{1} \mapsto y_{2} \mapsto-y_{1}, \quad y_{3} \mapsto y_{4} \mapsto-y_{3}, \\
& \tau: y_{1} \mapsto-y_{1}, \quad y_{2} \mapsto-y_{2}, \quad y_{3} \mapsto y_{3}, \quad y_{4} \mapsto y_{4}, \\
& \lambda: y_{1} \mapsto-y_{1}, \quad y_{2} \mapsto y_{2}, \quad y_{3} \mapsto-y_{3}, \quad y_{4} \mapsto-y_{4} .
\end{aligned}
$$

Hence $K\left(x_{1}, \ldots, x_{4}\right)^{\langle\tau\rangle}=K\left(y_{1}, \ldots, y_{4}\right)^{\langle\tau\rangle}=K\left(z_{1}, \ldots, z_{4}\right)$ where $z_{1}=y_{1}^{2}$, $z_{2}=y_{1} y_{2}, z_{3}=y_{3}, z_{4}=y_{4}$. Moreover, it can be verified that

$$
\begin{aligned}
& \sigma: z_{1} \mapsto z_{2}^{2} / z_{1}, \quad z_{2} \mapsto-z_{2}, \quad z_{3} \mapsto z_{4} \mapsto-z_{3}, \\
& \lambda: z_{1} \mapsto z_{1}, \quad z_{2} \mapsto-z_{2}, \quad z_{3} \mapsto-z_{3}, \quad z_{4} \mapsto-z_{4} .
\end{aligned}
$$

Define $u_{1}=z_{1}, u_{2}=z_{2}^{2}, u_{3}=z_{2} z_{3}, u_{4}=z_{3} z_{4}$. Then $K\left(z_{1}, \ldots, z_{4}\right)^{\langle\lambda\rangle}=$ $K\left(u_{1}, \ldots, u_{4}\right)$ and we find

$$
\sigma: u_{1} \mapsto u_{2} / u_{1}, \quad u_{2} \mapsto u_{2}, \quad u_{3} \mapsto-u_{2} u_{4} / u_{3}, \quad u_{4} \mapsto-u_{4}
$$

It is easy to check that $K\left(u_{1}, \ldots, u_{4}\right)^{\left\langle\sigma^{2}\right\rangle}=K\left(u_{1}, u_{2}, u_{3}^{2}, u_{4}\right)$.

Define

$$
v_{1}=u_{2}, \quad v_{2}=\left(u_{1}-\left(u_{2} / u_{1}\right)\right) u_{4}, \quad x=u_{1}, \quad y=u_{3}^{2} / u_{4} .
$$


We find that

$$
\sigma: v_{1} \mapsto v_{1}, \quad v_{2} \mapsto v_{2}, \quad x \mapsto v_{1} / x, \quad y \mapsto-v_{1} / y .
$$

By Theorem 2.2, $K\left(v_{1}, v_{2}, v_{3}, v_{4}\right)^{\langle\sigma\rangle}$ is rational over $K$.

Case 6. The group (II): $G=\left\langle\sigma, \tau: \sigma^{8}=\tau^{4}=1, \sigma^{4}=\tau^{2}, \tau^{-1} \sigma \tau=\sigma^{-1}\right\rangle$ and assume that $K\left(\zeta_{8}\right)$ is cyclic over $K$.

If $\zeta_{8} \in K$, then $K(G)$ is rational over $K$ by [Chu and Kang 2001, Theorem 1.6]. Hence we shall assume that $\zeta_{8} \notin K$ from now on.

Because $K\left(\zeta_{8}\right)$ is cyclic over $K$, it follows that $\operatorname{Gal}\left(K\left(\zeta_{8}\right) / K\right)=\langle\rho\rangle$ where $\rho\left(\zeta_{8}\right)=\zeta_{8}^{a}$ with $a=3,5$ or 7 .

We will find a faithful $G$-subspace $\bigoplus_{1 \leq i \leq 8} K \cdot x_{i}$ of $W=\bigoplus_{g \in G} K \cdot x(g)$ by

$$
\begin{aligned}
& x_{1}=x(1)-x\left(\sigma^{4}\right), \quad x_{i}=\sigma^{i-1} x_{1} \text { for } 2 \leq i \leq 4, \\
& x_{j}=\tau \sigma^{j-5} x_{1} \text { for } 5 \leq j \leq 8 .
\end{aligned}
$$

We find that

$$
\begin{aligned}
\sigma: x_{1} & \mapsto x_{2} \mapsto x_{3} \mapsto x_{4} \mapsto-x_{1}, \quad x_{8} \mapsto x_{7} \mapsto x_{6} \mapsto x_{5} \mapsto-x_{8}, \\
\tau: x_{1} & \mapsto x_{5}, \quad x_{2} \mapsto x_{6}, \quad x_{3} \mapsto x_{7}, \quad x_{4} \mapsto x_{8}, \quad x_{5} \mapsto-x_{1}, \quad x_{6} \mapsto-x_{2}, \\
x_{7} & \mapsto-x_{3}, \quad x_{8} \mapsto-x_{4} .
\end{aligned}
$$

We shall write $\zeta$ for $\zeta_{8}$ in the sequel and remember $\rho(\zeta)=\zeta^{a}$ with $a=3,5$ or 7. We shall extend the actions of $\sigma, \tau, \rho$ to $K(\zeta)\left(x_{1}, \ldots, x_{8}\right)$ by requiring $\sigma(\zeta)=\tau(\zeta)=\zeta$ and $\rho\left(x_{i}\right)=x_{i}$ for $1 \leq i \leq 8$. It follows that

$$
\begin{aligned}
K\left(x_{1}, \ldots, x_{8}\right)^{\langle\sigma, \tau\rangle} & =\left\{K(\zeta)\left(x_{1}, \ldots, x_{8}\right)^{\langle\rho\rangle}\right\}^{\langle\sigma, \tau\rangle} \\
& =K(\zeta)\left(x_{1}, \ldots, x_{8}\right)^{\langle\sigma, \tau, \rho\rangle} .
\end{aligned}
$$

Define $y_{1}, y_{3}, y_{5}, y_{7}, z_{1}, z_{3}, z_{5}, z_{7}$ by

$$
\begin{aligned}
& y_{1}=\left(\sigma-\zeta^{3}\right)\left(\sigma-\zeta^{5}\right)\left(\sigma-\zeta^{7}\right) \cdot x_{1}, \quad y_{3}=(\sigma-\zeta)\left(\sigma-\zeta^{5}\right)\left(\sigma-\zeta^{7}\right) \cdot x_{1} \\
& y_{5}=(\sigma-\zeta)\left(\sigma-\zeta^{3}\right)\left(\sigma-\zeta^{7}\right) \cdot x_{1}, \quad y_{7}=(\sigma-\zeta)\left(\sigma-\zeta^{3}\right)\left(\sigma-\zeta^{5}\right) \cdot x_{1} \\
& z_{1}=\left(\sigma-\zeta^{3}\right)\left(\sigma-\zeta^{5}\right)\left(\sigma-\zeta^{7}\right) \cdot x_{8}, \quad z_{3}=(\sigma-\zeta)\left(\sigma-\zeta^{5}\right)\left(\sigma-\zeta^{7}\right) \cdot x_{8} \\
& z_{5}=(\sigma-\zeta)\left(\sigma-\zeta^{3}\right)\left(\sigma-\zeta^{7}\right) \cdot x_{8}, \quad z_{7}=(\sigma-\zeta)\left(\sigma-\zeta^{3}\right)\left(\sigma-\zeta^{5}\right) \cdot x_{8} .
\end{aligned}
$$

It follows that

$$
\begin{aligned}
\sigma: y_{i} & \mapsto \zeta^{i} y_{i}, \quad z_{i} \mapsto \zeta^{i} z_{i} \text { for } i=1,3,5,7, \\
\tau: y_{1} & \mapsto-\zeta^{7} z_{7}, \quad y_{3} \mapsto-\zeta^{5} z_{5}, \quad y_{5} \mapsto-\zeta^{3} z_{3}, \quad y_{7} \mapsto-\zeta z_{1}, \\
z_{1} & \mapsto \zeta^{7} y_{7}, \quad z_{3} \mapsto \zeta^{5} y_{5}, \quad z_{5} \mapsto \zeta^{3} y_{3}, \quad z_{7} \mapsto \zeta y_{1} .
\end{aligned}
$$


If $\rho(\zeta)=\zeta^{a}$, then $\rho\left(y_{i}\right)=y_{a i}$ and $\rho\left(z_{i}\right)=z_{a i}$ for $i=1,3,5,7$. (The index $a i$ is understood to be modulo 8.)

Apply Theorem 2.1. Then

$$
K(\zeta)\left(x_{1}, \ldots, x_{8}\right)^{\langle\sigma, \tau, \rho\rangle}=K(\zeta)\left(y_{1}, y_{3}, y_{5}, y_{7}, z_{1}, z_{3}, z_{5}, z_{7}\right)^{\langle\sigma, \tau, \rho\rangle}
$$

is rational provided that $K(\zeta)\left(y_{1}, y_{3}, z_{5}, z_{7}\right)^{\langle\sigma, \tau, \rho\rangle}$ be rational when $\rho(\zeta)=\zeta^{3}$, that $K(\zeta)\left(y_{1}, y_{5}, z_{3}, z_{7}\right)^{\langle\sigma, \tau, \rho\rangle}$ be rational when $\rho(\zeta)=\zeta^{5}$, and $K(\zeta)\left(y_{1}, y_{7}, z_{1}, z_{7}\right)^{\langle\sigma, \tau, \rho\rangle}$ be rational when $\rho(\zeta)=\zeta^{7}$.

Subcase 6.1. $\rho(\zeta)=\zeta^{3}$.

Define

$$
u_{1}=y_{1} z_{7}, \quad u_{2}=y_{3} z_{5}, \quad u_{3}=y_{3} / y_{1}^{3}, \quad u_{4}=y_{1}^{8} .
$$

Then $K(\zeta)\left(y_{1}, y_{3}, z_{5}, z_{7}\right)^{\langle\sigma\rangle}=K(\zeta)\left(u_{1}, \ldots, u_{4}\right)$ and the actions of $\tau$ and $\rho$ are given by

$$
\begin{aligned}
& \tau: u_{1} \mapsto-u_{1}, \quad u_{2} \mapsto-u_{2}, \quad u_{3} \mapsto u_{2} / u_{1}^{3} u_{3}, \quad u_{4} \mapsto u_{1}^{8} / u_{4}, \\
& \rho: u_{1} \mapsto u_{2}, \quad u_{2} \mapsto u_{1}, \quad u_{3} \mapsto 1 /\left(u_{3}^{3} u_{4}\right), \quad u_{4} \mapsto u_{3}^{8} u_{4}^{3} .
\end{aligned}
$$

Define

$$
r=u_{1}, \quad s=u_{2} / u_{1}, \quad x=u_{1} u_{3}, \quad y=u_{3}^{2} u_{4} /\left(u_{1} u_{2}\right), \quad t=r(y-(1 / y)) .
$$

Then

$$
\begin{gathered}
\tau: r \mapsto-r, s \mapsto s, x \mapsto-s / x, y \mapsto 1 / y, t \mapsto t, \\
\rho: r \mapsto r s, s \mapsto 1 / s, x \mapsto 1 / x y, y \mapsto y, t \mapsto s t .
\end{gathered}
$$

Note that $K(\zeta)\left(u_{1}, \ldots, u_{4}\right)=K(\zeta)(r, s, x, y)=K(\zeta)(s, t, x, y)$.

By Theorem 2.2, $K(\zeta)(s, t, x, y)^{\langle\tau\rangle}=K(\zeta)(s, t, u, v)$ where

$$
u=\frac{x+\frac{s}{x}}{x y+\frac{s}{x y}}, \quad v=\frac{y-\frac{1}{y}}{x y+\frac{s}{x y}} .
$$

It is routine to check that $\rho(u)=1 / u, \rho(v)=s v / u$.

Define

$$
\begin{aligned}
& s^{\prime}=(\zeta-\rho(\zeta))(1+s)(1-s)^{-1}, \quad t^{\prime}=(1+s) t, \\
& u^{\prime}=(\zeta-\rho(\zeta))(1+u)(1-u)^{-1}, \quad v^{\prime}=(1+(s / u)) v .
\end{aligned}
$$

We find that $K(\zeta)(s, t, u, v)=K(\zeta)\left(s^{\prime}, t^{\prime}, u^{\prime}, v^{\prime}\right)$ and $\rho\left(s^{\prime}\right)=s^{\prime}, \rho\left(t^{\prime}\right)=t^{\prime}$, $\rho\left(u^{\prime}\right)=u^{\prime}, \rho\left(v^{\prime}\right)=v^{\prime}$. Thus $K(\zeta)(s, t, u, v)^{\langle\rho\rangle}=K\left(s^{\prime}, t^{\prime}, u^{\prime}, v^{\prime}\right)$ is rational.

Subcase 6.2. $\rho(\zeta)=\zeta^{5}$. 
Define

$$
u_{1}=y_{1} z_{7}, u_{2}=y_{5} z_{3}, u_{3}=y_{5} / y_{1}^{5}, u_{4}=y_{1}^{8} .
$$

Then $K(\zeta)\left(y_{1}, y_{5}, z_{3}, z_{7}\right)^{\langle\sigma\rangle}=K(\zeta)\left(u_{1}, \ldots, u_{4}\right)$ and the actions of $\tau$ and $\rho$ are given by

$$
\begin{aligned}
& \tau: u_{1} \mapsto-u_{1}, \quad u_{2} \mapsto-u_{2}, u_{3} \mapsto u_{2} / u_{1}^{5} u_{3}, u_{4} \mapsto u_{1}^{8} / u_{4}, \\
& \rho: u_{1} \mapsto u_{2}, u_{2} \mapsto u_{1}, u_{3} \mapsto 1 /\left(u_{3}^{5} u_{4}^{3}\right), u_{4} \mapsto u_{3}^{8} u_{4}^{5}
\end{aligned}
$$

Define

$$
r=u_{1}, s=u_{2} / u_{1}, x=u_{2}^{2} /\left(u_{3}^{3} u_{4}^{2}\right), \quad y=u_{1} u_{3}^{2} u_{4} / u_{2}, t=r(y-(1 / y)) .
$$

Then

$$
\begin{aligned}
& \tau: r \mapsto-r, s \mapsto s, x \mapsto s / x, y \mapsto 1 / y, t \mapsto t, \\
& \rho: r \mapsto r s, s \mapsto 1 / s, x \mapsto x y / s, y \mapsto 1 / y, t \mapsto-s t .
\end{aligned}
$$

By Theorem 2.2, $K(\zeta)\left(u_{1}, \ldots, u_{4}\right)^{\langle\tau\rangle}=K(\zeta)(s, t, x, y)^{\langle\tau\rangle}=K(\zeta)(s, t, u, v)$, where

$$
u=\frac{x-\frac{s}{x}}{x y-\frac{s}{x y}}, \quad v=\frac{y-\frac{1}{y}}{x y-\frac{s}{x y}} .
$$

It is routine to check that $\rho(u)=1 / u, \rho(v)=-s v / u$.

Define

$$
\begin{aligned}
& s^{\prime}=(\zeta-\rho(\zeta))(1+s)(1-s)^{-1}, \quad t^{\prime}=(1-s) t, \\
& u^{\prime}=(\zeta-\rho(\zeta))(1+u)(1-u)^{-1}, \quad v^{\prime}=(1-(s / u)) v .
\end{aligned}
$$

We find that $K(\zeta)(s, t, u, v)^{\langle\rho\rangle}=K\left(s^{\prime}, t^{\prime}, u^{\prime}, v^{\prime}\right)$ is rational.

Subcase 6.3. $\rho(\zeta)=\zeta^{7}$.

Define

$$
u_{1}=y_{1} z_{7}, u_{2}=y_{7} z_{1}, u_{3}=y_{7} / y_{1}^{7}, u_{4}=y_{1}^{8} .
$$

Then $K(\zeta)\left(y_{1}, y_{7}, z_{1}, z_{7}\right)^{\langle\sigma\rangle}=K(\zeta)\left(u_{1}, \ldots, u_{4}\right)$ and the actions of $\tau$ and $\rho$ are given by

$$
\begin{aligned}
& \tau: u_{1} \mapsto-u_{1}, u_{2} \mapsto-u_{2}, u_{3} \mapsto u_{2} / u_{1}^{7} u_{3}, u_{4} \mapsto u_{1}^{8} / u_{4}, \\
& \rho: u_{1} \mapsto u_{2}, u_{2} \mapsto u_{1}, u_{3} \mapsto 1 /\left(u_{3}^{7} u_{4}^{6}\right), u_{4} \mapsto u_{3}^{8} u_{4}^{7}
\end{aligned}
$$

Define

$$
r=u_{1}, s=u_{2} / u_{1}, x=u_{3} u_{4} / u_{1}, \quad y=u_{2}^{2} /\left(u_{1}^{2} u_{3}^{4} u_{4}^{3}\right), t=r(y-(1 / y)) .
$$


Then

$$
\begin{aligned}
& \tau: r \mapsto-r, s \mapsto s, x \mapsto-s / x, y \mapsto 1 / y, t \mapsto t, \\
& \rho: r \mapsto r s, s \mapsto 1 / s, x \mapsto x / s, y \mapsto 1 / y, t \mapsto-s t .
\end{aligned}
$$

By Theorem 2.2, $K(\zeta)\left(u_{1}, \ldots, u_{4}\right)^{\langle\tau\rangle}=K(\zeta)(s, t, x, y)^{\langle\tau\rangle}=K(\zeta)(s, t, u, v)$, where

$$
u=\frac{x-\frac{A}{x}}{x y-\frac{A B}{x y}}, \quad v=\frac{y-\frac{B}{y}}{x y-\frac{A B}{x y}}
$$

with $A=-s, B=1$.

It is routine to check that

$$
\rho: u \mapsto \frac{x-\frac{A}{x}}{\frac{B x}{y}-\frac{A y}{x}}, \quad v \mapsto \frac{-s\left(y-\frac{B}{y}\right)}{\frac{B x}{y}-\frac{A y}{x}} .
$$

Define $w=u / v$. Then $\rho(w)=-w / s$. Note that

$$
\frac{x-\frac{A}{x}}{\frac{B x}{y}-\frac{A y}{x}}=\frac{u}{b u^{2}-A v^{2}},
$$

because this is the same identity as the identity (3-1) we encountered in Case 1.

It follows that $\rho(u)=u /\left(B u^{2}-A v^{2}\right)=C / u$ where $C=w^{2} /\left(w^{2}+s\right)$.

Define

$$
p=(1-(1 / s)) w, \quad q=(1-s) t .
$$

Then $C=p^{2} /\left(s+(1 / s)+p^{2}-2\right)$.

It follows that $K(\zeta)(s, t, u, v)^{\langle\rho\rangle}=K(\zeta)(s, q, p, u)^{\langle\rho\rangle}$, where

$$
\rho: s \mapsto 1 / s, \quad q \mapsto q, \quad p \mapsto p, \quad u \mapsto C / u
$$

Define

$$
\begin{aligned}
& X=s+(1 / s), \quad Y=(\zeta-\rho(\zeta))(s-(1 / s)), \\
& Z=u+(C / u), \quad W=(\zeta-\rho(\zeta))(u-(C / u)) .
\end{aligned}
$$

Then $K(\zeta)(s, q, p, u)^{\langle\rho\rangle}=K(\zeta)(s, p, u)^{\langle\rho\rangle}(q)=K(X, Y, Z, W, p, q)$ because $[K(X, Y, Z, W, p)(\zeta): K(X, Y, Z, W, p)] \leq 2$. The relations of $X, Y, Z, W, p$ are given by

$$
1 X^{2}-(Y /(\zeta-\rho(\zeta)))^{2}=4
$$


and

(3-3) $Z^{2}-(W /(\zeta-\rho(\zeta)))^{2}=4 C=4 p^{2} /(s+(1 / s)+p-2)=4 p^{2} /\left(X+p^{2}-2\right)$.

Define $\eta=1 /(\zeta-\rho(\zeta))^{2} \in K$. Then we find, from (3-2), that $(X-2) / Y=$ $\eta Y /(X+2)$. From this we find that $(X-2) / Y, X$ and $Y$ all lie in $K((X+2) / Y)$.

Simplify (3-3). We get

$$
\left(Z\left(X-p^{2}-2\right) /(2 p)\right)^{2}-\eta\left(W\left(X-p^{2}-2\right) /(2 p)\right)^{2}=X+p^{2}-2 .
$$

Let $Z_{1}=Z\left(X-p^{2}-2\right) /(2 p)-p, Z_{2}=Z\left(X-p^{2}-2\right) /(2 p)+p$, and $W_{1}=$ $W\left(X-p^{2}-2\right) /(2 p)$. Thus (3-4) becomes $Z_{1} Z_{2}-\eta W_{1}^{2}=X-2 \in K((X+2) / Y)$. Thus $Z_{2}, p \in K\left((X+2) / Y, Z_{1}, W_{1}\right)$; hence $K(X, Y, Z, W, p, q)=K((X+2) / Y$, $\left.Z_{1}, W_{1}, q\right)$ is rational over $K$.

\section{References}

[Black 1999] E. V. Black, "Deformations of dihedral 2-group extensions of fields", Trans. Amer. Math. Soc. 351:8 (1999), 3229-3241. MR 99m:12004 Zbl 0931.12005

[Bogomolov 1987] F. A. Bogomolov, "The Brauer group of quotient spaces of linear group actions", Izv. Akad. Nauk SSSR Ser. Mat. 51:3 (1987), 485-516, 688. In Russian; translation in Math. USSR Izv. 30:3 (1988), 455-485. MR 88m:16006 Zbl 0679.14025

[Chu and Kang 2001] H. Chu and M.-c. Kang, "Rationality of p-group actions", J. Algebra 237:2 (2001), 673-690. MR 2001k:13008 Zbl 1023.13007

[Chu et al. 2004] H. Chu, S.-J. Hu, and M.-c. Kang, "Noether's problem for dihedral 2-groups", Comment. Math. Helv. 79:1 (2004), 147-159. MR 2004i:12004 Zbl 02055141

[DeMeyer and McKenzie 2003] F. DeMeyer and T. McKenzie, "On generic polynomials", J. Algebra 261:2 (2003), 327-333. MR 2003m:12007 Zbl 1021.12004

[Furtwängler 1925] P. Furtwängler, “Über Minimalbasen für Körper rationalen Funktionen”, Sitzungsber. Akad. Wiss. Wien 134 (1925), 69-80.

[Garibaldi et al. 2003] S. Garibaldi, A. Merkurjev, and J.-P. Serre, Cohomological invariants in Galois cohomology, University Lecture Series 28, American Mathematical Society, Providence, RI, 2003. MR 2004f: 11034 Zbl 01959122

[Gröbner 1934] W. Gröbner, "Minimalbasis der Quaternionengruppe”, Monatshefte Math. Phys. 41 (1934), 78-84.

[Haeuslein 1971] G. K. Haeuslein, "On the invariants of finite groups having an abelian normal subgroup of prime index”, J. London Math. Soc. (2) 3 (1971), 355-360. MR 43 \#3241 Zbl 0213.31002

[Hajja 1983] M. Hajja, "Rational invariants of meta-abelian groups of linear automorphisms", $J$. Algebra 80:2 (1983), 295-305. MR 84g:20010 Zbl 0544.20007

[Hajja and Kang 1994] M. Hajja and M.-c. Kang, "Three-dimensional purely monomial group actions”, J. Algebra 170:3 (1994), 805-860. MR 95k:12008 Zbl 0831.14003

[Hajja and Kang 1995] M. Hajja and M.-c. Kang, "Some actions of symmetric groups", J. Algebra 177:2 (1995), 511-535. MR 96i:20013 Zbl 0837.20054

[Hashimoto and Miyake 1999] K.-I. Hashimoto and K. Miyake, "Inverse Galois problem for dihedral groups", pp. 165-181 in Number theory and its applications (Kyoto, 1997), edited by S. 
Kanemitsu and K. Györy, Dev. Math. 2, Kluwer Acad. Publ., Dordrecht, 1999. MR 2001a:12010 Zbl 0965.12004

[Huppert 1967] B. Huppert, Endliche Gruppen. I, Grundlehren der Mathematischen Wissenschaften 134, Springer, Berlin, 1967. MR 37 \#302 Zbl 0217.07201

[Kang 2004] M.-c. Kang, "Introduction to Noether's problem for dihedral groups", Algebra Colloq. 11:1 (2004), 71-78. MR 2058965 Zbl 1061.12005

[Kemper 1996] G. Kemper, “A constructive approach to Noether's problem”, Manuscripta Math. 90:3 (1996), 343-363. MR 97d:13005 Zbl 0865.12005

[Kemper and Malle 1999] G. Kemper and G. Malle, "Invariant rings and fields of finite groups", pp. 265-281 in Algorithmic algebra and number theory (Heidelberg, 1997), edited by B. H. Matzat et al., Springer, Berlin, 1999. MR 99m:13010 Zbl 0934.13002

[Kervaire and Vust 1989] M. Kervaire and T. Vust, "Fractions rationnelles invariantes par un groupe fini: quelques exemples", pp. 157-179 in Algebraic Transformation groups and invariant theory, edited by H. Kraft et al., DMV Sem. 13, Birkhäuser, Basel, 1989. MR 1044591 Zbl 0703.14006

[Kuniyoshi 1955] H. Kuniyoshi, “On a problem of Chevalley”, Nagoya Math. J. 8 (1955), 65-67. MR 16,993d Zbl 0065.02602

[Ledet 2000] A. Ledet, "Generic polynomials for quasi-dihedral, dihedral and modular extensions of order 16”, Proc. Amer. Math. Soc. 128:8 (2000), 2213-2222. MR 2000k:12003 Zbl 0952.12002

[Ledet 2001] A. Ledet, "Generic polynomials for $Q_{8^{-}}, Q C$-, and $Q Q$-extensions", J. Algebra 237:1 (2001), 1-13. MR 2001k:12012 Zbl 1004.12002

[Lenstra 1974] H. W. Lenstra, Jr., "Rational functions invariant under a finite abelian group", Invent. Math. 25 (1974), 299-325. MR 50 \#289 Zbl 0292.20010

[Maeda 1989] T. Maeda, "Noether's problem for A5", J. Algebra 125:2 (1989), 418-430. MR 91c: 12004 Zbl 0697.12018

[Miyata 1971] T. Miyata, "Invariants of certain groups, I", Nagoya Math. J. 41 (1971), 69-73. MR 42 \#7804 Zbl 0211.06801

[Saltman 1982] D. J. Saltman, "Generic Galois extensions and problems in field theory", Adv. in Math. 43:3 (1982), 250-283. MR 84a:13007 Zbl 0484.12004

[Saltman 1984] D. J. Saltman, "Noether's problem over an algebraically closed field", Invent. Math. 77:1 (1984), 71-84. MR 85m:13006 Zbl 0546.14014

[Serre 1995] J.-P. Serre, "Résumé des cours de 1993-1994", pp. 91-98 in Annuaire du Collège de France (1994), 1995. Reprinted as pp. 435-442 in Euvres = Collected papers, vol. IV: 1985-1998, Springer, Berlin, 2000. MR 2001e:01037 Zbl 0933.01034

[Swan 1983] R. G. Swan, "Noether's problem in Galois theory", pp. 21-40 in Emmy Noether in Bryn Mawr (Bryn Mawr, PA, 1982), edited by B. Srinivasan and J. Sally, Springer, New York, 1983. MR 84k:12013 Zbl 0538.12012

Received to be supplied.

MING-CHANG KANG

DEPARTMENT OF MATHEMATICS

NATIONAL TAIWAN UNIVERSITY

TAIPEI

TAIWAN

kang@math.ntu.edu.tw 